\title{
Introduction to the HICSS-55 Minitrack on Location Intelligence for System Sciences Research
}

\author{
James B. Pick \\ University of Redlands \\ James_Pick@redlands.edu
}

\section{Introduction to Minitrack}

This mini-track includes scholarly research papers that apply a variety of theories and empirical techniques, quantitative, and qualitative, to understand how location, geography, and related data are important to incorporate into the system sciences. Some of the papers develop new theories or modify existing ones for the systems sciences that incorporate spatial data, locational intelligence, and geographical concepts. Current ideas of data science, analytics algorithms, clustering, risk analysis, and optimization can be brought to bear on addressing locational intelligence research questions. Some of the investigations focus on important practical problems, such as appraising real estate, optimally locating bus stops, understanding geographic clusters of high drug abuse, and mitigating fire-prone landscapes, while others concern spatial analytics algorithms and geometric theory underpinning location intelligence. The papers are grouped into two areas of location intelligence and analytics: section 1, which describes and prescribes solutions to emergencies, and section 2, which emphasizes novel techniques of location intelligence and geospatial analysis. The sections are followed by a conclusion.

\section{Location analytics approaches to mitigate emergencies}

The increasing interest in this HICSS minitrack during the past year may stem in part from the covid-19 pandemic, which as a global emergency has required locational intelligence solutions. For instance, the famous location intelligence model has been the Covid 19 Dashboard of Center for Systems Science and Engineering at Johns Hopkins University (CSSE, 2021). In contrast to that dashboard's amazing big data management structure with limited location analytics, the four emergency-related papers in this minitrack employ smaller data sets, but apply more powerful location intelligence techniques, to address pressing problems of drug use and environment, rather than covid-19.

The four contributions apply a variety of techniques

\author{
Avijit Sarkar \\ University of Redlands \\ Avijit_Sarkar@redlands.edu
}

and theories, which draw on GIScience, optimization, and spatial statistics.

The paper, "Geospatial Clustering Analysis on Drug Abuse Emergencies,” by J. Lee, J.I. Choi, B.-Y. Yeh, Q. Lan, and H. Kang, develops and tests a geospatial model to estimate spatial clusters based on demographic and socio-economic variables, utilizing a machine learning algorithm for clustering. The study practically addresses the very serious opioid drug abuse emergency, with particular focus on the state of Ohio. The data are drawn from emergency-medical-service (EMS) dispatch records for the city of Cincinnati. The data on heroin incidence as well as geographical variables and socioeconomic and demographic co-variates are geo-referenced by block group, cleaned, formatted and integrated.

An unsupervised machine learning cluster algorithm was used to define Cincinnati's clusters of heroin addiction based on EMS data. THE K-means clustering method yields six clusters, which are characterized by 20 co-variates. Using random-forest regression, the covariates are ranked in importance of contribution to heroin overdose incidence, revealing population density, downtown zoning, percent male, and manufacturing zoning as positively related, while education, age, and diversity are inversely associated. The findings are interpreted and explained. An important implication for healthcare planning and monitoring is that areas of high racial/ethnic diversity for Cincinnati are likely to imply low heroin-overdose incidence.

In many parts of the world, deadly wildfires are becoming more commonplace and connection of such wildfires to climate change has been the topic of much recent research. Some of this research uses GIS and location analytical approaches. The paper "Location Analytics for Transitioning to Fire Resilient Landscapes," by A. Murray, R.L. Church, B.A. Pludow, and P. Stine is an important and timely contribution. In this paper, the authors propose an analytical approach to transition landscapes to being more fire resilient. The approach combines GIS and location analytics with mathematical optimization modeling. Specifically, the authors amend the knapsack problem formulation to incorporate spatial contiguity constraints to identify the best land units to treat 
in a forest in order to reduce the threat of wildfire, but doing so while honoring budget constraints. While the formulated model's objective function is exactly similar to the knapsack problem, the model's novelty comes from new and unique set of decision variables and constraints which track contiguity as a network flow process to ensure that any selected land unit is connected to neighboring selected units. This novel yet elegant modeling is one of the paper's distinguishing features.

The model is tested for 582 land management units spread over 7,557 acres of the Stanislaus National Forest which consists of nearly 900,000 acres in the Sierra Nevada mountains of California. Without spatial contiguity, the knapsack model suggested the selection of 36 land units with a total benefit of 87.52 and a solution time of 1 second. However, when spatial contiguity constraints are added, the amended model selected 23 contiguous land units totaling 100 acres for treatment with a potential benefit of almost 47. By imposing spatial contiguity, added benefit is a casualty, yet there are operational efficiencies for the U.S. Forest Service, which can readily move treatment equipment and personnel between units, eliminating the need for more costly transportation and set-up services. Overall, this paper showcases the power of combining location analytics and spatial optimization to solve a real-world problem of great importance.

The paper "Evacuation Shelter Scheduling Problem" by H. Shimizu and co-authors examines the problem of scheduling evacuation shelters that become necessary in the event of large-scale disasters. This is also a timely contribution especially when environmental and other man-made disasters have caused untold misery in many parts of the world. The bigger a disaster, the costlier it becomes to operate evacuation shelters. When the number of evacuees decreases, they are often relocated to reduce costs, yet this imposes an emotional toll on the impacted evacuees.

The authors formulate the evacuation shelter scheduling problem as a quadratic programming optimization model, which is unwieldy. When solved using a commercial solver, the solution time is prohibitive. To overcome this issue, the authors formulated a $0-1$ integer programming relaxation that can be solved optimally by appropriately setting the movement and operation costs. Test results of the model for an event resembling the Kobe City earthquake in Kobe, Japan were promising. Simulation experiments found optimal locations of evacuation shelters in nine wards of Kobe City along with trajectories of evacuees relocated from location to another. Overall, the model's performance was strong and reduced operation costs by 33.7 million dollars, or $32 \%$ savings in operational costs, compared to before. Both the Murray et al. and Shimizu et al. papers show the potency of combining GIS and location analytics with optimization modeling. While the extent of integration of GIS and optimization was different in the two papers, they illustrate a systems science approach to societal and environmental problems of significant importance.

More precisely, author propose an optimization model for allocating evacuees to shelters in such a way that the cost to move evacuees from one shelter to another as well as the operational costs of shelters are minimized. The efficacy of the proposed model is tested for the study area affected by the Kobe earthquake in Japan. Experiments show that the proposed method, that is, its integer programming transformation yields significant savings in operational costs of shelters.

Underpinned by indoor GIS, indoor navigation and indoor positioning systems are increasing in prominence in many sectors and industries. They are particularly relevant for emergency evacuation of those trapped indoors during emergencies. The paper "Location intelligence system for people estimation in indoor environment during emergency operation" by F. Fallucchi and co-authors proposes a simple, low-cost locationintelligence based emergency evacuation in an indoor environment.

The authors design a system based on conditions that do not necessarily assume that individuals are carrying location-sensing devices such as cell phones. The proposed system is based on a deep neural network (DNN) model that detects people within rooms/buildings using wireless channel state information. The test system, data collection and manipulation processes are described along with the DNN model and experimental results. The performance of the DNN model is compared to several well-known machine learning techniques. Tests showed that the defined neural network model in many cases performed better than traditional machine learning models. The model behaved well even with signals collected in environments different from the training one. Additional testing in different sized rooms would be necessary for further validation of the proposed model.

\section{Novel methods for location intelligence}

The paper, "Using Geolocated Text to Quantify Location in Real Estate Appraisal," by T. Heuwinkel, J.P. Kucklick, and O. Muller, offers an enhanced method to predict real estate appraisals that utilizes text data from geo-referenced Wikipedia articles that are text analyzed to enhance conventional methods. The text-analytic approach goes beyond the ordinary model input of the object's position, to add characteristics contextual information for each object. To convert textual information to assist machine learning (ML), the text is transformed for use in the model by ensemble learning based on gradient boosting, rather than the conventional regression approach. 
The new method is tested on how effective Wikipedia articles are for appraising properties in Allegheny County, Pennsylvania, based on data about real estate, place, and Wikipedia text. Data were filtered to include important POIs, removing lesser ones. The text was represented is a new "spatially weighted TF-IDF approach" to create vectors for input into ML. Details are given on this method. The new TF-IDF approach in the ML model performed better than traditional GIS-based features by $8.2 \%$. The authors point to future research using deep learning techniques or improving interpretability by latent Dirichlet allocation.

The paper, "A Method for the Optimized Placement of Bus Stops Based on Voronoi Diagrams," by T. Stadler, S. Hofmeister, and J. Dunnweber, focuses on a novel methodology to locate bus stops. A method using Voronoi diagrams enables the constrained placement of bus stops so that the stops are more in areas of dense population and less in low-population areas. The Voronoi algorithm seeks to address the "first mile problem," i.e. by locating bus stops so that commuting from home to the bus stop is reduced. The solution also takes into account two types of rural areas, one with bus stops already assigned and another in which there are no bus stops or only a few.

A Voronoi diagram consists of the partitioning of a plane that consists of convex polygons with its generating point that, for each point in a single polygon, is the closest generating point. The Voronoi diagram and the extended Delaunay triangulation can be partitioned into subpolygons, which, combined with settlement and other variables, determine location of new bus stops. The paper concentrates on explaining the set of detailed steps and algorithms that generate the new bus stops.

The model was tested for the small town of Roding, Germany including surrounding towns and villages for 2,200 new bus stops. Run time metrics were produced. The large number of stops may partly be explained as regulatory placeholders that are common in Europe to allow passengers to get on or off. The Voronoi model is compared to other models, showing that it can be effective with fewer parameters, while taking into account all the business firms in the area.

The paper, "Assessing Multi-Agent Reinforcement Learning Algorithms for Autonomous Sensor Resource Management," by T.J. Bihl, A. Jones, K.. Straub, B. Bontempo, and F. Jones, compares methods and algorithms to achieve reinforcement learning through management of sensor resources for a general location intelligence routing task. The study is applied to unmanned aerial vehicles (UAVs), in particular to UAV search and rescue operations, based on artificial intelligence. Sensor resource management combined with location intelligence allows the UAVs, in conjunction with their sensors, to complete a task. Yet there is a challenge, which is to gain operational autonomy. Multi- agent reinforcement learning (MARL) algorithms are potentially able to help achieve the autonomy in sensor resource management.

The optimization of parameters was compared for nine MARL algorithms. Each algorithm was tested through a hyperparameter search, which produced parameters to train superior models. Value-based algorithms (Ape-X with $\mathrm{DQN}$ ) gave the best performance. In second place, the policy gradient (PG) algorithm was found to perform comparatively well versus the other algorithms. A tradeoff between target reward and episode length is noted for the RAINBOW algorithm, which did well at finding its target, but did so slowly. The other algorithms have problems in performance, poor locating of target, or low training stability. SAC had the best performance in the group of actor-critic algorithms.

\section{Conclusion}

Location analytics has branched into multiple disciplinary areas. It has adopted theories and methods from analytics, GIScience, information systems, and computer science, so it truly crosses over many areas of the systems sciences.

The papers in the minitrack emphasize a variety of approaches, methods, and findings for location analytics in emergency situations. This includes a set of four papers -- for the terrible spread of heroin overdoses, for locating evacuation shelters for example during the Kobe earthquake in Japan, for making the changes to mitigate of fire potential through fire resistant landscapes in the US, and location intelligence applied to rescue in indoor emergencies. Although these papers use different methods including cluster analysis, machine learning, principal components analysis, spatial optimization, and spatial contiguity, the theme running through them is to better prepare for, and mitigate potential disasters.

A second set of papers concern novel approaches to location analytics. One study offers the innovation of incorporating geolocated text on points of interest to enhance the prediction of real estate values. The text processing provides a distillation of knowledge about points of interest that enriches prediction of pricing beyond ordinary real estate indicators. A second investigation on optimizing the placement of bus stops innovates by using the geometric properties of Voronoi diagrams to partition the land area under study, incorporate some other variables, and improve the identification of bus stops for populations that have reduced access to bus stops. The third study presents a novel system for sensor resource management that can be applied to multiple unmanned aerial vehicles based on the AI method of multi-agent reinforcement learning. It should be pointed out that the four emergency papers also have novel aspects. 
Altogether, the papers in the minitrack add to the knowledge and practice of location analytics and intelligence in the system sciences. They also exemplify that much is still unknown. This is indicative of the potential for novel applications of real-world importance and future research and knowledge discovery in this area.

\section{Reference}

CSSE, "COVD-19 Dashboard," Baltimore, Maryland: Center for Systems Sciences and Engineering, Johns Hopkins, University, 2021. 